\title{
ANDES

www.scielo.cl

\section{Confiabilidad inter e intraobservador de la escala BITSS en el diagnóstico y seguimiento precoz de la diarrea infecciosa en niños menores de 3 años}

\author{
Inter- and intra-observer reliability of BITSS scale in the diagnosis and \\ early follow-up of infectious diarrhea in children under 3 years of age
}

Edgar Játiva-Mariño ${ }^{\mathrm{a}, \mathrm{b}}$, Carlos Manterola ${ }^{\mathrm{b}, \mathrm{c}}$, Tamara Otzen $^{\mathrm{b}, \mathrm{c}}$

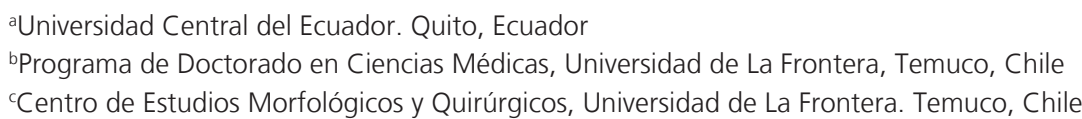

Recibido: 11 de mayo de 2021; Aceptado: 30 de julio de 2021

\section{¿Qué se sabe del tema que trata este estudio?}

La escala BITTS, es un instrumento de medición de reciente desarrollo, de la cual existe escasa información, pues se encuentra en procesos de validación y estudios de confiabilidad.

\section{¿Qué aporta este estudio a lo ya conocido?}

El estudio de confiabilidad inter e intra observador de la escala BITTS.

\section{Resumen}

La escala BITSS (Brussels Infant and Toddler Stool Scale), es un instrumento utilizado en diagnóstico y seguimiento de bebés y niños pequeños con diarrea infecciosa. Consta de 7 fotografías de pañales con distintos tipos de heces. Objetivo: Determinar confiabilidad inter e intra observador de la escala BITSS en lactantes y niños que utilizan pañal. Pacientes y Método: Estudio de confiabilidad. Se reclutaron tres pediatras con experiencia en tratamiento de diarrea infecciosa, a los que previo entrenamiento en aplicación de escala BITSS, se les solicitó evaluar 70 fotografías de deposiciones obtenidas de pacientes pediátricos que representan los 7 diferentes tipos de heces que reconoce la escala (10 imágenes por ítem). Dos pediatras participaron en el estudio de confiabilidad interobservador, ambos evaluaron de forma enmascarada las mismas fotografías, asignado un valor a cada cual, y el tercero en la evaluación intra-observador, evaluando 70 imágenes en dos oportunidades distintas, separadas 15 días. Se determinó grado de acuerdo entre observadores y estadística kappa; para la escala y cada ítem de la escala. Resultados: Se realizó un total de 280 mediciones, 140 para el estudio
Palabras clave: Diarrea Infantil; Reproducibilidad; Escala de Heces; Escala BITSS

Correspondencia:

Carlos Manterola

carlos.manterola@ufrontera.cl 
de confiabilidad inter observador y 140 para el de confiabilidad intra observador. Confiabilidad inter observador: el grado de acuerdo entre observadores para la escala fue $85.7 \%$; con un kappa de 0.817 (IC 95\% de 0.72; 0.92), con un error estándar de 0.05. Confiabilidad intra observador: El kappa intra observador para la escala, fue de 0.883 (IC $95 \%$ de 0.80 ; 0.96 ), con un error estándar de 0.04 . Conclusión: La escala BITSS tiene buena confiabilidad intra e interobservador.

\begin{abstract}
The BITSS scale (Brussels Infant and Toddler Stool Scale) is an instrument used in the diagnosis and follow-up of infants and young children with infectious diarrhea. It consists of 7 pictures of diapers with different types of stools. Objective: To determine intra and interobserver reliability of the BITSS scale in infants and children using diapers. Patients and Method: Reliability study. Three pediatricians with experience in treating childhood infectious diarrhea were selected and, after training in applying the BITSS scale, they evaluated 70 pictures of stools obtained from pediatric patients, representing the 7 different types of stools categorized by the scale ( 10 images for each item). Two pediatricians participated in the interobserver reliability study and performed a blind evaluation of the same pictures, assigning a value to each one, and the third pediatrician participated in the intra-observer analysis, evaluating 70 images on two different occasions, 15 days apart. Interobserver agreement and kappa statistics were determined for the scale and each scale item. Results: 280 measurements were made, 140 for the inter-observer reliability study and 140 for the intra-observer one. The degree of inter-observer agreement for the scale was $85.7 \%$; with a kappa of 0.817 (95\% CI $0.72 ; 0.92)$, and standard error of 0.05 ; and the intra-observer kappa for the scale was 0.883 (95\% CI 0.80; 0.96), with a standard error of 0.04 . Conclusions: The BITSS scale has good intra- and interobserver reliability.
\end{abstract}

\section{Keywords:}

Infectious Diarrhea;

Reproducibility of

Results;

Stool Scale;

BITSS Scale

\section{Introducción}

Se estima que en sus primeros 5 años de vida, aproximadamente 17 de cada 100 niños sufre un episodio de diarrea aguda, en 9 de ellos se busca atención médica, uno de cada 20 necesitan hospitalización y uno de cada 375 fallecen. En el registro del Instituto Nacional de Estadística y Censos del 2017 se verificó que entre las causas de mortalidad en menores de 5 años la enfermedad diarreica aguda infantil representa el 2,6\% ${ }^{1}$.

La diarrea infecciosa aguda sigue siendo una importante carga de enfermedad en todo el mundo, especialmente en países en desarrollo. El principal problema es la deshidratación secundaria y el objetivo terapéutico es mejorar y mantener el estado de hidratación ${ }^{2,3}$. Las manifestaciones clínicas y el estado general del niño deshidratado forman parte de la evaluación de la enfermedad, pero uno de los pilares del diagnóstico y la evolución de los pacientes es la consistencia de las deposiciones ${ }^{3}$. Con base en este hecho, el 2009 se desarrolló y validó la escala Amsterdam Infant Stool Scale (AISS), para valorar consistencia, volumen y color de las heces de niños menores de 1 año ${ }^{4}$, sin embargo, en la práctica clínica y la investigación pediátrica no se utiliza de forma habitual.

Posiblemente en respuesta a lo anterior, en 2017, se desarrolló la escala BITSS (Brussels Infant and Toddler Stool Scale $)^{5}$, herramienta de valoración visual de heces adaptada para bebés y niños pequeños que usan pañales, que consta de 7 fotografías en color de pañales que contienen heces de bebés y niños pequeños no entrenados para ir al baño (figura 1). Esta escala fue sometida a un proceso de validación de contenido, comparándola con la escala BSFS (Bristol Stool Form Scale $)^{6}$.

Por otra parte, es necesario conocer lo válido y confiable que puede ser una medición obtenida con un instrumento de medición. En este sentido, es relevante comprender que la confiabilidad, reproducibilidad o consistencia de las mediciones de una escala es una característica fundamental de la mediciones que se pretenden realizar con ella, puesto que entre otras cosas, permitirá la eventual comparabilidad de resultados en diversos escenarios, momentos y poblaciones si la escala se aplica en las mismas condiciones ${ }^{7}$.

El objetivo de este estudio fue determinar confiabilidad inter e intra observador de la escala BITSS en lactantes y niños no entrenados a ir al baño y que utilizan pañal.

\section{Pacientes y Método}

El informe de este estudio se realizó siguiendo la guía GRRAS, para el reporte de estudios de confiabilidad $^{8}$. 


\section{Diseño}

Estudio de confiabilidad.

\section{Definiciones}

Se definió como confiabilidad inter observador, al grado de acuerdo obtenido entre dos investigadores independientes al aplicar la escala BITSS a las mismas fotografías $(\mathrm{n}=70)$ en un mismo período de tiempo. Se definió como confiabilidad intra observador, al grado de acuerdo obtenido por el mismo investigador al aplicar la escala BITSS a las mismas fotografías $(n=70)$, en dos momentos diferentes, con un intervalo de 15 días entre una medición y otra ${ }^{7}$.

\section{Tamaño de la muestra}

Se utilizó el criterio de factibilidad propuesto por Streiner, de 10 mediciones por cada ítem de la escala ${ }^{9}$. Como la escala BITSS está compuesta por 7 ítems (fotografías que muestran diferentes tipos de deposiciones), se consideró que la muestra mínima era de 70 fotografías a evaluar. Para ello, se recolectaron 180 diferentes fotografías de heces de niños lactantes y menores de 3 años no entrenados para ir al baño; a partir de las cuales, se seleccionaron las 10 más representativas de conformidad a las características visuales para cada tipo de deposición definida por la escala BITSS.

\section{Evaluadores}

Se seleccionaron tres médicos especialistas en pe- diatría. Todos, con experiencia en diagnóstico y tratamiento de enfermedad diarreica infantil (ML, RM y $\mathrm{RQ})$.

\section{Proceso de medición}

Los médicos pediatras fueron capacitados de forma virtual, para visualizar el tipo y consistencia de heces de conformidad a los grados de la escala BITSS (Figura 1), con 3 fotografías por tipo de deposiciones. Una vez finalizado el entrenamiento, a dos de ellos (ML, RM); se les envío de forma simultánea e independiente, una carpeta con las 70 fotografías organizadas al azar para estudiar confiabilidad ínter observador. A la otra pediatra (RQ), se le envió un set con 70 fotografías al azar para su diagnóstico; y luego de 15 días, se le remitió nuevamente la misma serie de 70 fotografías, para determinar confiabilidad intra observador.

Los datos de las 4 mediciones fueron recogidas de forma codificada y enmascarada, en una planilla Excel.

\section{Análisis estadístico}

Después de realizar un análisis exploratorio de los datos se aplicó estadística descriptiva. Posteriormente se realizó determinación de la confiabilidad inter observador e intra observador mediante grado de acuerdo entre observadores y estadística kappa ${ }^{10}$; para la escala y para cada ítem de la escala. Se utilizaron como estadísticas complementarias para corroborar resultados, las pruebas Tau-b de Kendall y Gamma.

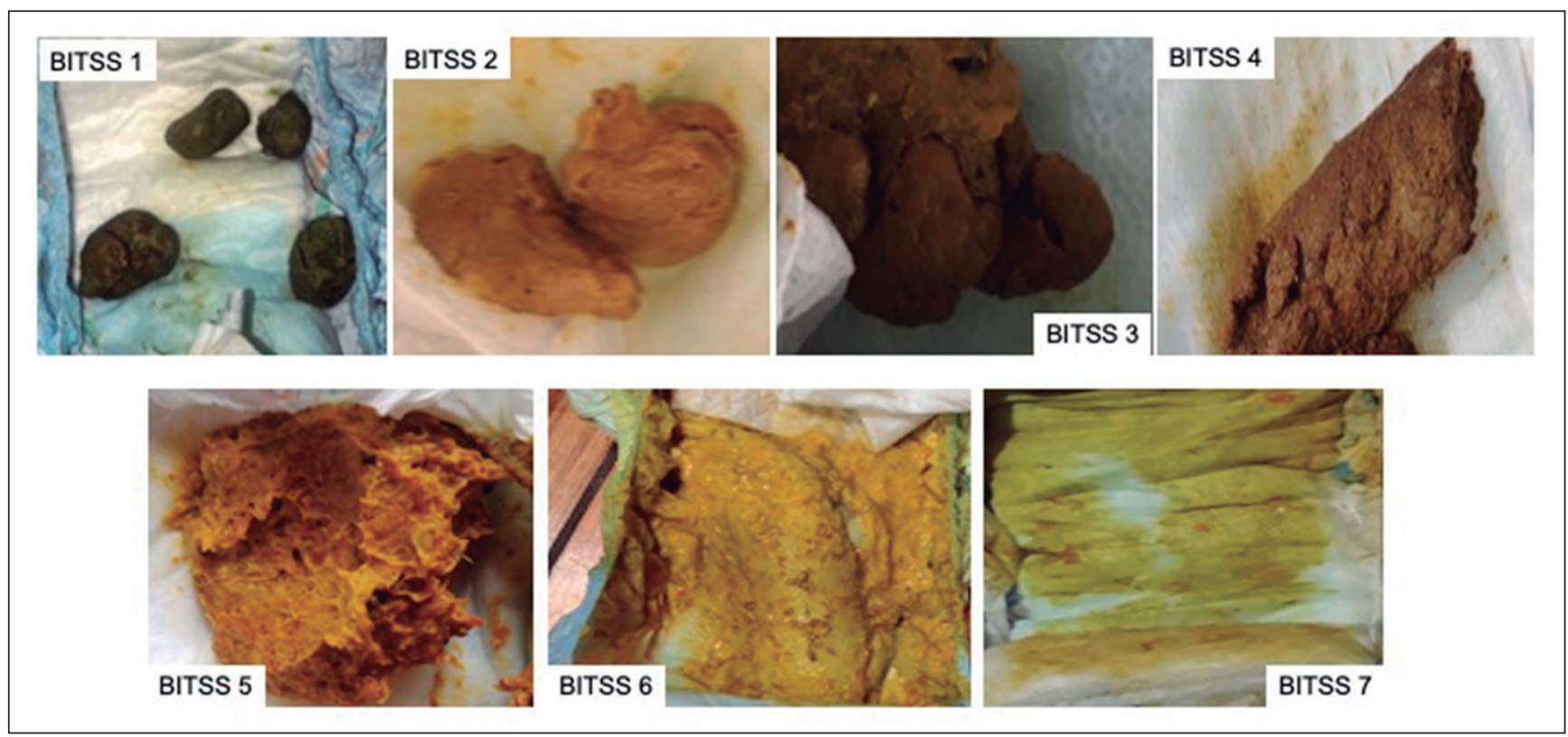

Figura 1. Imágenes de los diferentes tipos de heces clasificadas en la escala BITSS. BITSS 1. Heces duras. Bultos duros separados, como nueces. BITSS 2. Heces duras. En forma de salchicha pero abultada. BITSS 3. Heces duras. Como una salchicha pero con grietas en su superficie. BITSS 4. Heces formadas. Como una salchicha o una serpiente, lisa y suave. BITSS 5. Heces blandas. Suaves con bordes bien definidos. BITSS 6. Heces blandas. Piezas esponjosas con bordes irregulares. BITSS 7. Heces líquidas, acuosas, sin estructuras sólidas. 


\section{Aspectos éticos}

Se resguardó la confidencialidad de los pacientes a partir de los cuales se capturaron las imágenes de sus pañales; y se observaron los principios de la declaración de Helsinki ${ }^{11}$.

\section{Resultados}

Se realizó un total de 280 mediciones, 140 para el estudio de confiabilidad inter observador y 140 para el de confiabilidad intra observador.

Los médicos pediatras que participaron como observadores son dos mujeres y un hombre, con una mediana de edad de 51 años. Dos de ellos, son médicos del hospital pediátrico Baca Ortiz de Quito y la otra, pertenece a la Seguridad Social. Todos tienen una experiencia profesional de 14 años como pediatras y 20.6 años de médicos. Dos de ellos desconocían absolutamente la escala BITSS y uno de ellos tenía referencias teóricas, pero no la había utilizado aún.

\section{Confiabilidad inter observador}

El grado de acuerdo entre observadores para la escala fue 85,7\%; con un kappa de 0,817 (IC 95\% de $0,72 ; 0,92)$, con un error estándar de 0,05 . El detalle del kappa para cada ítem de la escala, se puede apreciar en la tabla 1.

\section{Confiabilidad intra observador}

El kappa intra observador para la escala, fue de 0,883 (IC 95\% de 0,80; 0,96), con un error estándar de 0,04 . El detalle del kappa para cada ítem de la escala, se puede apreciar en la tabla 1.

\section{Discusión}

Cada día se hace más necesario contar con instrumentos válidos y confiables para realizar mediciones en clínica, así como para conducir estudios. En este caso, siendo la enfermedad diarreica aguda infantil y su principal complicación la deshidratación expresada en deposiciones líquidas (que cambian de conformidad a la gravedad y evoluticidad de la enfermedad), nos pareció de interés aportar en el proceso de validación de la escala BITSS ${ }^{6,12}$, para diagnóstico y seguimiento precoz de la diarrea infecciosa en niños menores de 3 años, generando resultados de confiabilidad interobservador e intraobservador de la escala, aplicada por pediatras generales.

Es por ello que consideramos que es un aporte al conocimiento del médico pediatra, que puede contar con una escala para diagnóstico y seguimiento precoz de la diarrea infecciosa infantil, la que puede evolucionar desde el ítem 7 a 6 o 5 en la valoración, representando mejoría y buen pronóstico, y de ahí a 4 o menos hasta la mejoría completa del cuadro, o por el contrario, cuadros que inician en 5 y pueden evolucionar a 7, representando un curso tórpido y eventual aparición de complicaciones.

Al planificar el estudio, constatamos que la aplicación del instrumento requería de un proceso de calibración entre los usuarios; de lo contrario, se corría el riesgo de tener criterios de medición e interpretación distintos y por ende resultados dispares, lo que constituiría un error sistemático en su aplicación y por tanto, un sesgo de medición.

Pudimos verificar que la escala BITSS como constructo, presenta un buen comportamiento de confiabilidad inter e intraobservador, y al valorar cada uno de los 7 ítems por separado, verificamos que en todas

Tabla 1. Valores de la estadística kappa y complementarias Tau-b de Kendall y Gamma; para confiabilidad inter e intra observador de la escala BITSS

\begin{tabular}{|c|c|c|c|c|c|c|}
\hline & $\begin{array}{c}\text { Kappa } \\
\text { Inter observador }\end{array}$ & IC 95\% & Error estándar & $\begin{array}{c}\text { Kappa } \\
\text { Intra observador }\end{array}$ & IC 95\% & Error estándar \\
\hline BITSS 1 & 1,0 & --- & --- & 1,0 & --- & --- \\
\hline BITSS 2 & 0,89 & $0,73-1$ & 0,08 & 0,70 & $0,11-1$ & 0,30 \\
\hline BITSS 3 & 1,0 & --- & --- & 1,0 & --- & --- \\
\hline BITSS 4 & 1,0 & --- & --- & 0,93 & $0,85-1$ & 0,04 \\
\hline BITSS 5 & 0,61 & $0-1$ & 0,34 & 1,0 & --- & --- \\
\hline BITSS 6 & 1,0 & --- & --- & 1,0 & --- & --- \\
\hline BITSS 7 & 1,0 & --- & --- & 1,0 & --- & --- \\
\hline Escala total & 0,817 & 0,$72 ; 0,92$ & 0,05 & 0,883 & 0,$8 ; 0,96$ & 0,04 \\
\hline
\end{tabular}

IC: Intervalo de confianza del $95 \%{ }^{15}$. 
las mediciones con excepción de una (inter observador BITSS 5) los valores fueron superiores a 0,7 , e incluso algunas fueron superiores a 0,9 , lo que se interpreta como valores con fuerza de concordancia "buenos" y "muy buenos"7. No obstante ello, en la valoración de los ítems por separado, se aprecia que los errores de los observadores fueron siempre de confusión con el ítem inmediatamente anterior o posterior, lo que podría deberse a cierta similitud en las imágenes. Es así como en la evaluación intraobservador se constató problemas entre los ítem 2 y 3 y entre los ítems 3 y 4 . Por otra parte, en la evaluación ínter observador, ocurrió algo similar entre los ítems 5 y 6 . Estos errores, pueden deberse a calidad insuficiente de las imágenes, entrenamiento insuficiente de los observadores, o a cierta similitud entre algunos ítems que harían plantear la posibilidad de reducir los ítems de la escala fusionando alguno (s) de ellos; lo que puede ser motivo de un nuevo estudio.

Respecto de limitaciones del estudio, se pueden mencionar que los observadores evaluaron imágenes de forma digital, modo que podría asociarse a cierto riesgo de sesgo de medición. Por otra parte, nos parece que el tiempo de capacitación de los observadores pudo influir en los resultados finales, de tal forma que para reproducir estos resultados es necesario contar con un tiempo mínimo de entrenamiento, así como un número de imágenes por cada ítem de la escala, de forma tal de lograr fijar en la memoria las imágenes de los distintos tipos de deposiciones identificadas por la escala BITSS ${ }^{13}$.

Nos parece interesante comentar también, que estos resultados permitirán continuar trabajando con la aplicabilidad de la escala BITSS (a modo de línea de investigación), en otras entidades clínicas de la práctica pediátrica de lactantes y menores de 3 años que utilizan pañal; como algunas disfunciones gastrointestinales y el estreñimiento infantil, dadoque en estas patologías, la valoración de la consistencia de las heces es fundamental ${ }^{14}$.

Del mismo modo, nos parece que a continuación (conociendo la confiabilidad de la escala en personal entrenado), corresponde su aplicación por parte de médicos generales, enfermeras, padres, tutores, etc.; de forma tal de ir extendiendo su uso, para posteriormente realizar estudios de confiabilidad en este tipo de observadores. Esta línea de trabajo permitirá contar con una herramienta simple, posiblemente confiable en sus mediciones, y de uso generalizado más allá del especialista entrenado, en escenarios como los servicios de urgencias.

En resumen, se puede concluir señalando que la escala BITSS tiene muy buena confiabilidad inter observador e intra observador. Por lo que puede ser considerada como una escala a utilizar en la práctica clínica pediátrica, por ser una instrumento diagnóstico de fácil aplicación y reproducible.

\section{Responsabilidades Éticas}

Protección de personas y animales: Los autores declaran que los procedimientos seguidos se conformaron a las normas éticas del comité de experimentación humana responsable y de acuerdo con la Asociación Médica Mundial y la Declaración de Helsinki.

Confidencialidad de los datos: Los autores declaran que han seguido los protocolos de su centro de trabajo sobre la publicación de datos de pacientes.

\section{Derecho a la privacidad y consentimiento informa-} do: Los autores han obtenido el consentimiento informado de los pacientes y/o sujetos referidos en el artículo. Este documento obra en poder del autor de correspondencia.

\section{Conflicto de intereses}

Los autores declaran no tener conflicto de intereses.

\section{Referencias}

1. Castillo C, Fuentes DE, Ballesteros C Taco C. Instituto Nacional de Estadística y Censos. Informe de rendición de cuentas. Período: enero - diciembre 2017. Disponible en: https://www. ecuadorencifras.gob.ec/documentos/ web-inec/Rendicion_de_cuentas_2017/ Fase_3/Informe $\% 20$ tecnico $\% 20$ Planta\%20Central\%20RC\%20 2017(final)10FEB2018.pdf.

2. Florez ID, Niño-Serna LF, BeltránArroyave CP. Acute Infectious Diarrhea and Gastroenteritis in Children. Curr Infect Dis Rep. 2020;22:4.

3. Játiva-Mariño E, Manterola C, Macias R, Narváez D. Probióticos y prebióticos. Rol en la terapéutica de la enfermedad diarreica aguda infantil. Int J Morphol. 2021;39:294-301.

4. Bekkali N, Hamers SL, Reitsma JB, et al. Infant Stool Form Scale: Development and Results. J Pediatr. 2009;154: 521-6.e1.

5. Vandenplas Y, Szajewska H, Benninga $\mathrm{M}$, et al. Development of the Brussels Infant and Toddler Stool Scale ('BITSS'):
Protocol of the study. BMJ Open. 2017;7:2016-8

6. Velasco-Benitez CA, Llanos-Chea A, Saps M. Utility of the Brussels Infant and Toddler Stool Scale (BITSS) and Bristol Stool Scale in non-toilet-trained children: A large comparative study. Neurogastroenterol Motil. 2020;e14015.

7. Manterola C, Grande L, Otzen T, et al. Reliability, precision or reproducibility of the measurements. Methods of assessment, utility and applications in clinical practice. Rev Chilena Infectol. 2018;35:680-8. 
8. Kottner J, Audigé L, Brorson S, et al. Guidelines for reporting reliability and agreement studies (GRRAS) were proposed. J Clin Epidemiol. 2011;64:96106.

9. Streiner DL., Norman GR. Health measurement scales: a practical guide to their development and use. $4^{\text {th }}$ edition. Oxford, UK: Oxford University Press; 2008.

10. Ubersax J. Kappa Coefficients. Statistical Methods for Diagnostic Agreement 2010 update. Disponible en http://johnuebersax.com/stat/kappa2.htm.

11. Helsinki Statement. WMA Declaration of
Helsinki - Ethical Principles for Medical Research Involving Human Subjects. 64th WMA General Assembly, Fortaleza, Brazil, October 2013. Disponible en: https://www.wma.net/policies-post/wmadeclaration-of-helsinki-ethical-principlesfor-medical-research-involving-humansubjects.

12. Huysentruyt K, Koppen I, Benninga M, et al. The Brussels Infant and Toddler Stool Scale: A Study on Interobserver Reliability. J Pediatr Gastroenterol Nutr. 2019;68:207-13.

13. Játiva-Mariño E, Rivera-Valenzuela MG, Velasco-Benitez CA, Saps M. The prevalence of functional constipation in children was unchanged after the Rome IV criteria halved the diagnosis period in Rome III. Acta Paediatr. 2019;108:2274-7.

14. Játiva-Mariño E, Játiva-Cabezas Z, Velasco-Benítez CA. Prevalencia de desórdenes gastrointestinales funcionales y hábito intestinal en lactantes menores de 12 meses internados en el Hospital Infantil Baca Ortíz de Quito, Ecuador. MÉD UIS. 2019;32:13-21.

15. Dominguez-Lara SA. Confidence interval for Kappa coefficient in Sánchez-Sánchez et al. Enferm Intensiva. 2016;27:132. 